\title{
Multidisciplinary Design Study of Heavy Vechical Rupd Crashworthiness And Energy Absorption
}

\author{
Alok Kumar Khore ${ }^{1}$, Tapan Jain $^{2}$, Kartikeya Tripathi ${ }^{3}$ \\ 1. Lecturer, Mechanical Department, Sanghvi Institute of Management \& Science. Indore, \\ 2. Associate Professor, Acropolis Institute of Technology \& Research, Indore, \\ Research Scholar Suresh Gyan Vihar University Jaipur \\ 3. Professor, Mechanical Engineering Department, SGSITS, Indore
}

\begin{abstract}
The paper focuses on simulation \& analysis of a Rear Under Run Protection (RUPD) system under crash scenario. The basic objective is to improve the safety of the car and the occupants by designing the RUPD and car bumper. The choice of material and the structural design are the two major factors for impact energy absorption during a crash. The deformation of RUPD bar and plastic strains in RUPD components can be determined before the physical test for predicting failure of the system to meet the compliance requirements as per IS 14812-2005. Additionally, failure of the RUPD attachment points with chassis can be determined. Physical testing can be reduced significantly with this approach which ultimately reduces the total cycle time as well as the cost involved in product development. This project explains the FE analysis of RUPD for impact loading. All the results obtained from the impact load analysis are evaluated against the requirements of IS 14812-2005 which could reduce the process development time and cost involved in the same.

The analysis was carrying out using Finite Elements software (LS-Dyna), Meshing tools by Altair Hyper mesh and Modeling on Pro-E. This analysis is a partial work of a major project wherein the RUPD will be subjected to static testing with variable load distributions at different locations on RUPD. The analysis establishes the method and parameters of the simulation on modeling and analysis software used by demonstrating the energy absorption pattern in bumper and RUPD during frontal crash of a car with different design parameters of RUPD.
\end{abstract}

Index Terms: - CAD(Modeling \& Simulation-Pro-E), Meshing (Hypermesh), Preprocessing (LS-Dyna), ANSYS solver.

\section{INTRODUCTION}

The collisions can be classified in many ways such as crashes oncoming vehicle's lane, under icy, snowy, or wet conditions; crashes into heavy vehicles generally occurred in daylight, on workdays, in winter etc. Primary evaluation is according to head and chest injuries. The injuries are categorized based on critical, death head injuries and multiple fatal injuries. Investigators also looked at data concerning suicide and driving with alcohol for a proper statistical representation. They also observed that the risk of frontal collisions may be reduced by a mid RUPD, front energy absorbing structure for trucks and buses and driving conditions.

The accidental event, when a passenger car or a light load-carrying vehicle crashes and is wedged under the rear part of the vehicle chassis, is called rear under run. The rear under run protection device (RUPD) prevents the vehicles from being wedged under the chassis during accidental crashes and with that significantly increases the safety of occupants. This necessitates the requirement of conscious a proper design. The most important condition is the RUPD resistance to loading forces acting along or parallel to the vehicle longitudinal axis. The regulation also calls for a practical RUPD testing on the testing machine, where the RUPD is subjected to prescribed loads at some particular loading points. If the measured deformations fall into the allowable range, the RUPD can be declared to comply with the regulation. The practical testing is required for all standard mounted RUPD.

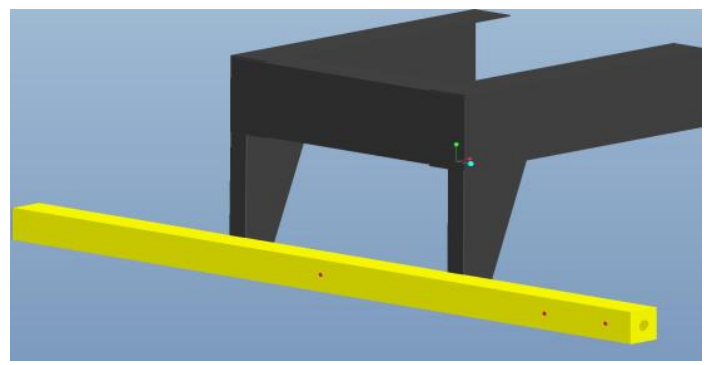

Fig 1.1 RUPD Design 1 


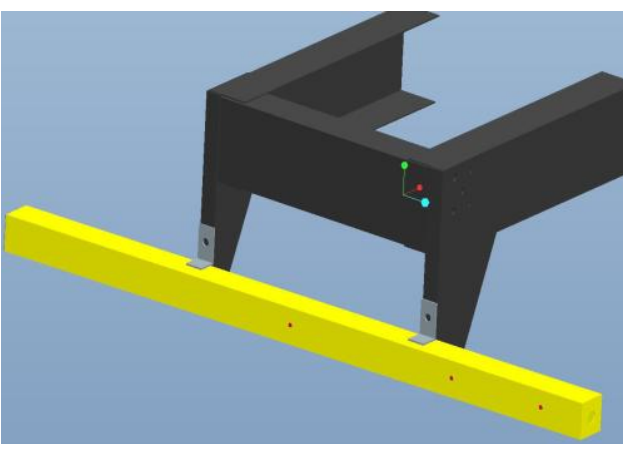

Fig 1.2 RUPD Design 2

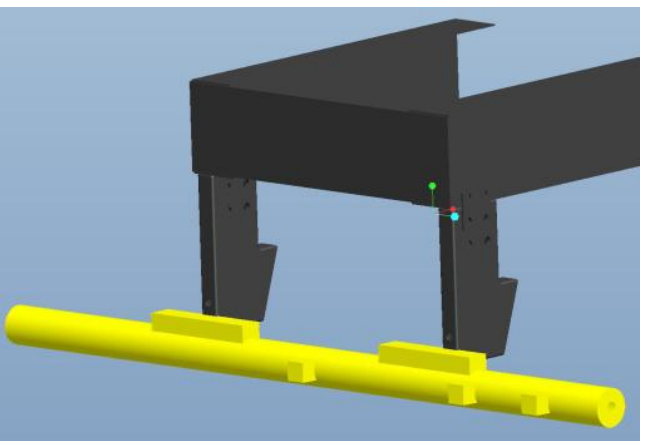

Fig 1.3 RUPD Design 3

Heavy commercial vehicle under run Protection (URP) has a long history of investigation. European research organizations as well as heavy commercial vehicle manufacturers have been studying the subject since the 80s, initially commencing with rear and side URP and followed by front URP. Research in Australia, Canada, and the United States commenced in the late 90s and focused mainly on rear under runs, which in Australia contributes to only about 10 per cent of under run trauma. In recent years, the member countries of the European Union have been instrumental in financing and managing research efforts directed at generating solutions for addressing front under run trauma, which in Australia accounts for 75 per cent of under run trauma. Protection for vulnerable road users and passenger car occupants from heavy commercial vehicle under run is now mandatory in Europe for commercial vehicles exceeding a GVM of 3.5 tones. Some member countries of ASEAN and the three most populous and fast growing economies of China, India and Brazil also have some form of URP requirements for heavy commercial vehicles.

\section{SURVEY}

Road accidents are human tragedy. They involve high human suffering and monetary loss in terms of fatality, injuries and loss of potential income. During the calendar year 2010, there were close to 5 lakh road accidents in India, which resulted in more than 1.3 lakh deaths and injuries on 5.2 lakh persons. These numbers translate into one road accident every minute, and one road accident death every 4 minutes.

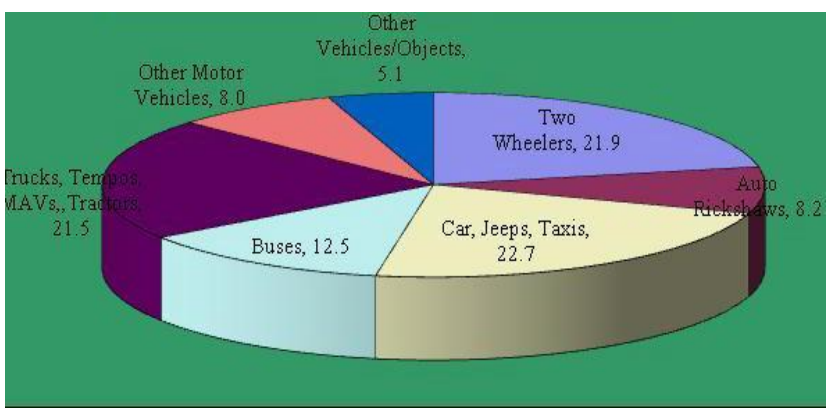

In 2010-2011 an accidental (Fig 1.2) shows maximum Percent of Trucks and car, jeep approx 21.5 to 22.7 its huge injuries cause of front and rear crash. Trucks accident results in major deaths because high impact and low safety equipments. It was observed in a survey at National highway 3 and National highway 59 that maximum accidents takes place on rear of truck because of rear view and improper mountings of RUPD.

\section{LEGAL REQUIREMENTS OF RUPD}

RUPDs to be implemented are regulated by ECE's R58. An Indian regulation IS 14812 - 2005 is derived from ECE R58 standard, 


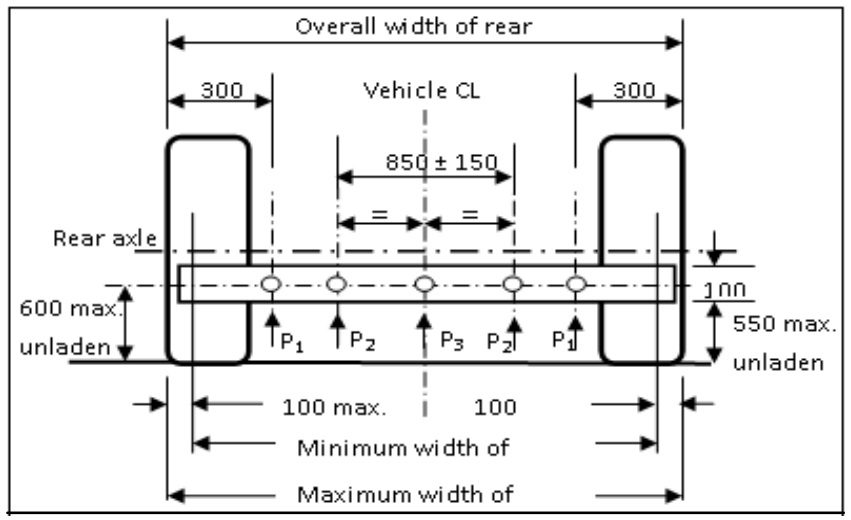

Fig 1.5 RUPD Requirement

1. The device shall offer adequate resistance to forces applied parallel to the longitudinal axis of the vehicle, and be connected; when in the service position with the chassis side members or whatever replaces them. This requirement shall be satisfied if it is shown that both during and after the application, the horizontal distance between the rear of the device and the rear extremity of the vehicle does not exceed $400 \mathrm{~mm}$ at any of the points P1, P2 and P3 (Fig.1.3). In measuring this distance, any part of the vehicle which is more than $3 \mathrm{~m}$ above the ground when the vehicle is un-laden shall be excluded. Point $P$, are located $300+25 \mathrm{~mm}$ from the longitudinal planes tangential to the outer edges of the wheels on the rear axle; point P2 which are located on the line joining point P1, are symmetrical to the median longitudinal plane of the vehicle at a distance from each other of 800 to $1100 \mathrm{~mm}$ inclusive, the exact position being specified by the manufacturer. The height above the ground of points P1, and P2 (see Figure 1.3) shall be defined by the vehicle manufacturer within the lines that bound the device horizontally. The height shall not, however, exceed $600 \mathrm{~mm}$ when the vehicle is un-laden. P3 is the centre point of the straight line joining point $\mathrm{P} 2$.

2. A horizontal force equal to 12.5 percent of the maximum technically permissible weight of the vehicle but not exceeding $25 \mathrm{KN}$ shall be applied successively to both points $\mathrm{P}$, and to point $\mathrm{P} 3$.

3. A horizontal force equal to 50 percent of the maximum technically permissible weight of the vehicle but not exceeding $100 \mathrm{KN}$ shall be applied successively to both points $\mathrm{P} 2$.

4. The forces specified above shall be applied separately, on the same guard. The order in which the forces are applied may be specified by the manufacturer.

5. Whenever a practical test is performed to verify compliance with the above mentioned requirements, the following conditions shall be fulfilled.

\section{METHODOLOGY}

In modern engineering analysis it is rare to find a project that does not require some type of simulation for analyzing the behavior of the model under certain specified conditions. The advantages of simulation are numerous and important. A new design concept may be modeled to determine its real world behavior under various load environments, and may therefore be refined prior to the creation of drawings and changes can be inexpensive. Once a detailed CAD model has been developed, simulations can analyze the design in detail, saving time and money by reducing the number of prototypes required. An existing product which is experiencing a field problem, or is simply being improved, can be analyzed to speed an engineering change and reduce its cost. This paper considered Design 1 point P3 for impact and energy absorbing area.

Crash-testing requires a number of the test vehicle to be destroyed during the course of the tests and is also time consuming and uneconomical. One new recent trend that is gaining vast popularity is computer simulated crash-testing. Here instead of a real vehicle, a FE (Finite Element) model of the vehicle is generated and is used to carry out the different tests that were carried out before using actual vehicles. There are several software packages that are equipped to handle the crash-testing of vehicles, but one of the most popular in dynamic analysis software is LS-DYNA.

In this analysis the rear impact crash is conducted using a modeled (car), and truck chassis (half segment) as the test Finite element model. The car and the RUPD were modeled on Pro-E and integrated on Hypermesh for critical meshing. After meshing the system, the model was imported in LS-Dyna environment (LS-Prepost) for setting various simulation parameters. The output of Prepost (.k file) was solved in ANSYS LS-Dyna solver. The truck chassis has a fixed and the initial velocity of car model is assumed $50 \mathrm{kmph}$ before impact the RUPD bar. The simulation is given a termination time $0.5 \mathrm{sec}$. The reason for termination time is that for rigid RUPD bar. 
The Model generated is in 5 steps:

1. Modeling (Pro-E)

2. Meshing (Altair Hyper mesh)

3. Pre-Processing

4. Solver

5. Post Processing

Pro/ENGINEER is a feature-based product development tool. The models are constructed using a series of easy to understand features rather than confusing mathematical shapes and entities. The geometric dentition of a model is defined by the type of features used and by the order in which each feature is placed. Each feature builds upon the previous feature and can reference any of the preceding features; this enables design intent to be built into the model. Individually, each feature is typically simple but as they are added together they form complex parts and assemblies. After selecting features, geometry, or components in a model, assembly, or drawing, you are able to make medications to the selected items. Direct selection is one of the three basic methods of selection.

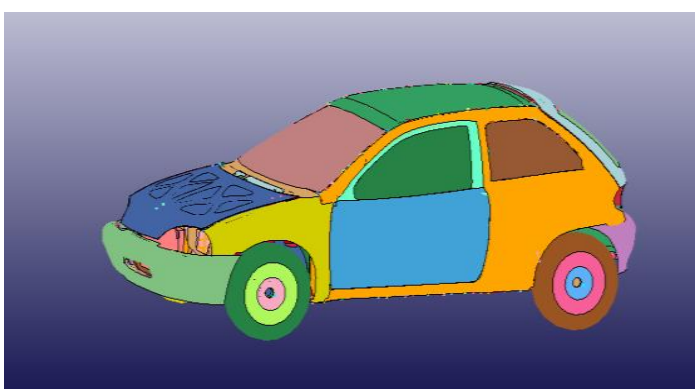

Fig 1.5 Car Design

\section{Hypermesh (Meshing )}

Universal finite element pre- and post-processor. Hypermesh is a high-performance finite element preand post-processor for major finite element solvers, which allows engineers to analyze design conditions in a highly interactive and visual environment. Hypermesh's user-interface is easy to learn and sup-ports the direct use of CAD geometry and existing finite element models, providing robust interoperability and efficiency. Advanced automation tools within Hypermesh allow users to optimize meshes from a set of quality criteria, change existing meshes through morphing, and generate mid-surfaces from models of varying thickness. Reduce time and engineering analysis cost through high-performance finite element modeling and post-processing and reduce redundancy and model development costs through the direct use of CAD geometry and existing finite element models and Support numerous commercial solvers by providing direct interfaces to a wide array of analysis codes ensuring the best code can be used for specific situations and also Cost-effective pricing to deliver maximum functionality for your software investment.

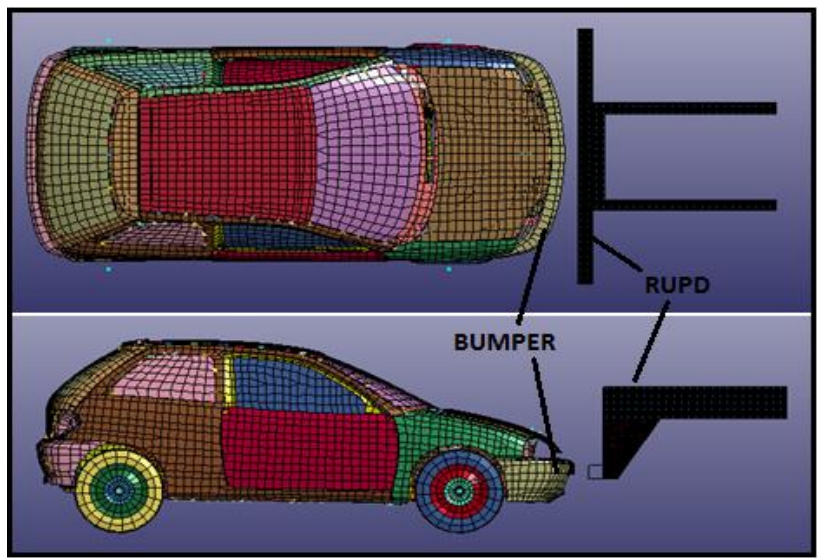

Fig :1.6 Car And RUPD Meshing

\section{ELEMENTS \& BOUNDARY CONDITIONS}

The completed model contains approximately 208 parts, 61 materials and 42191 elements and 45547 nodes. Structural components and specific element types used in the model include. 


\author{
1. Beam \\ 2. Discrete \\ 3. Mass \\ 4. Seatbelt accelerometer \\ 5. Shell \\ 6. Solid
}

The function of the boundary conditions is to create and define constraints and loads on finite element models. To simulate a full vehicle car crash all loads and boundary conditions that occur in the actual crash event need to be modeled. Just as a car is subjected to gravitational loads in real life, the simulated model should have a representative gravity force applied. Friction forces between the tires and the road surface play an important role in how the vehicle behaves on impact, so these have to be accounted for in the simulation.

The crash compatibility of the ULSAB with cars of similar weight and geometric characteristics is assessed using the results of computer numerical simulation of the crash scenario that depicts the frontal collision with full engagement (no offset) of RUPD and car, each with a forward velocity of $13.4 \mathrm{~m} / \mathrm{s}(35 \mathrm{mph})$. An important issue is the compatibility of vehicles. There could be adverse effects on vehicle fleet compatibility after structural changes. A vehicle which has a stiffer or more aggressive front structure for his own increased frontal safety could be more dangerous for another car, especially if that other car is involved in a front impact crash. Also the use of the same fixed deformable RUPD for light and heavy cars could lead to less compatibility in crashes between small and large cars. The amount of energy absorbed by the RUPD is for a light car a larger proportion of the total crash energy as for a heavy car. To achieve a level of performance comparable to a small car, the front structure of the large car must be designed to crush more or to crush at a higher force level to absorb the additional energy.

It is possible that a small car becomes softer because a lot of its energy was absorbed by the RUPD. This is another reason why a second rigid RUPD test is important. The increased crash velocity by India from $56 \mathrm{~km} / \mathrm{h}$ to $64 \mathrm{~km} / \mathrm{h}$ has also a negative influence on the compatibility. This velocity increase yields a 30 per cent higher amount of crash energy. That means that for the same deformation length the force level and thus the stiffness of all cars has to grow with 30 per cent. This effect increases the absolute difference in force levels between light and heavy cars, which deteriorates the compatibility. Otherwise the test velocity must be higher as where collision statistics ask for, because for a comparable vehicle deformation as in a car to car crash the initial kinetic energy must be higher to compensate the absorbed energy in the RUPD. Another interesting test for the compatibility problem is a test with a moving deformable RUPD. Such a test simulates much better collisions between cars and could improve the fleet compatibility.

In this case the smaller vehicle is subjected to a harsher crash environment due to the higher energy absorption and a higher velocity change yielding a stiffer structure. On the other hand the large car would be subjected to a less severe crash environment in terms of velocity change, so a softer front structure gives a temperate crash pulse.Crash analysis is very helpful for experts of road vehicle accidents, as well, since their work requires simulations and data, which are as close to the reality as possible. By developing the applied methods and algorithms we can make the simulations more precise and so contribute towards the determination of the factors causing the accident. Through the analysis of traffic accidents we can obtain information concerning the vehicle which can be of help in modifying the structure/parameters to improve its future safety.

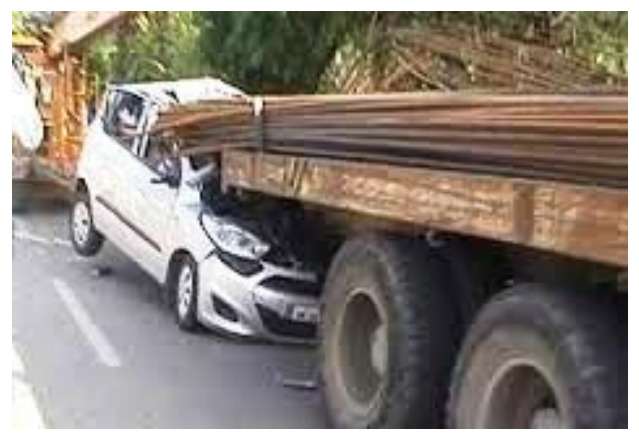

Fig : 1.7 Rear accident

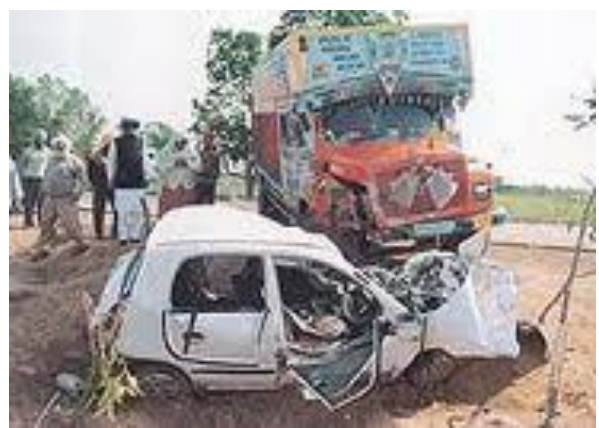

Fig : 1.8 front accident

Two additional concepts that govern motion apply to vehicle and occupant kinematics as well as occupant restraint systems. These are: Energy, which is the property (or capacity) of a body or a system that enables it to do work and overcome resistance, and Work, which is the transference of energy from one body to the other. Energy 
is an abstract concept, which cannot be seen, felt, tasted or smelled. The only time energy becomes evident is when it changes. Energy appears in many forms including thermal (heat), solar (light), electrical (electricity), and mechanical energies. The most commonly known energies are kinetic energy (K.E.) and potential energy (P.E.). In mechanical systems, a force does work on a body provided that the point where the force is applied has a component of displacement in the direction of force. The work done, or energy transferred to or from the body, is then equal to the magnitude of the force times the distance over which it acts. The kinetic energy, which is the energy of motion of a body, is equal to half its mass multiplied by its velocity squared as expressed by the following relationship:

$$
K . E .=(m . v 2) / 2
$$

where $m$ is the mass of the body and $v$ is the velocity. Potential energy is a stored energy within a body or a system because of its position with respect to other bodies. When energy is in the stored state, a body or a system has the potential for doing work.

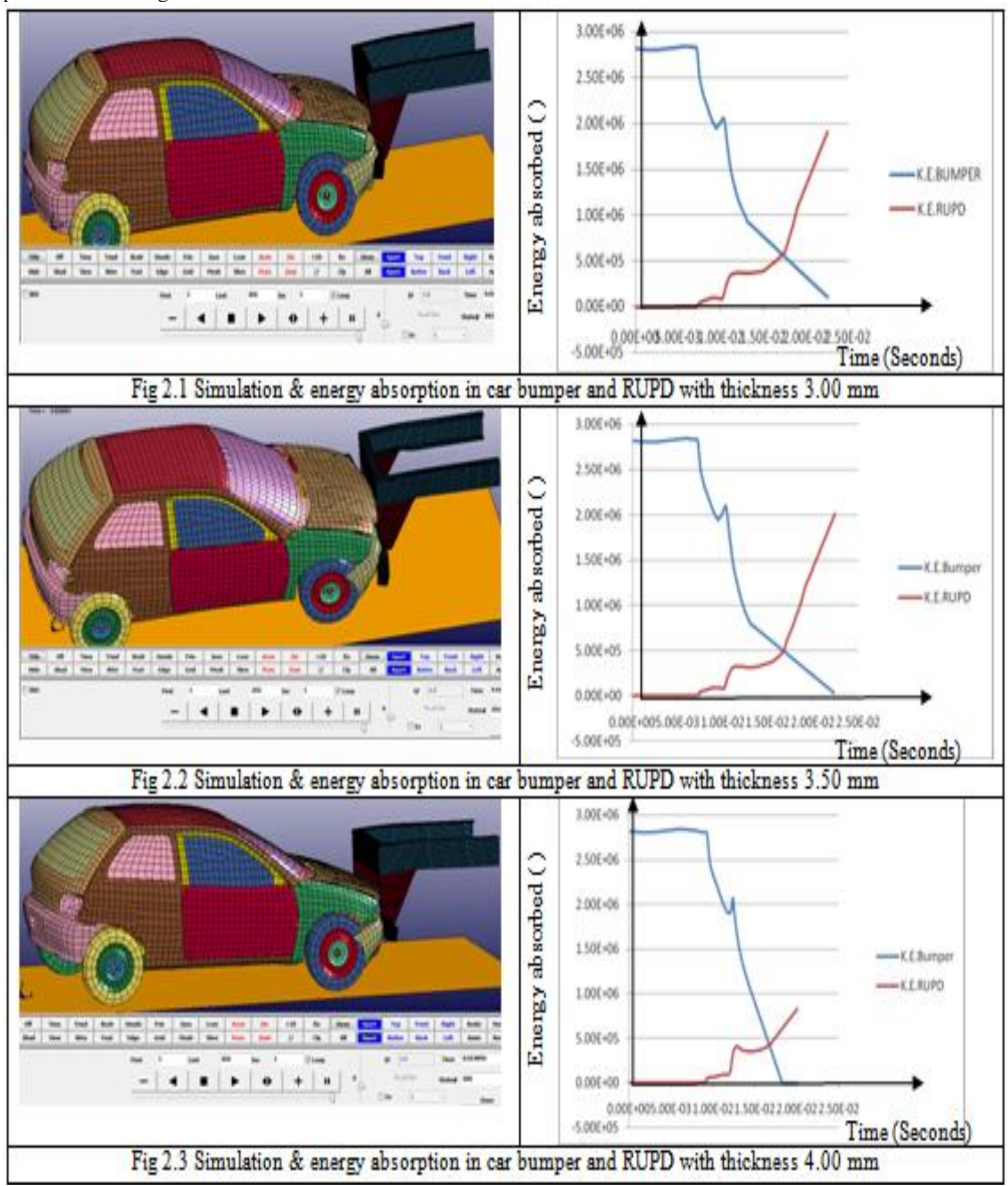



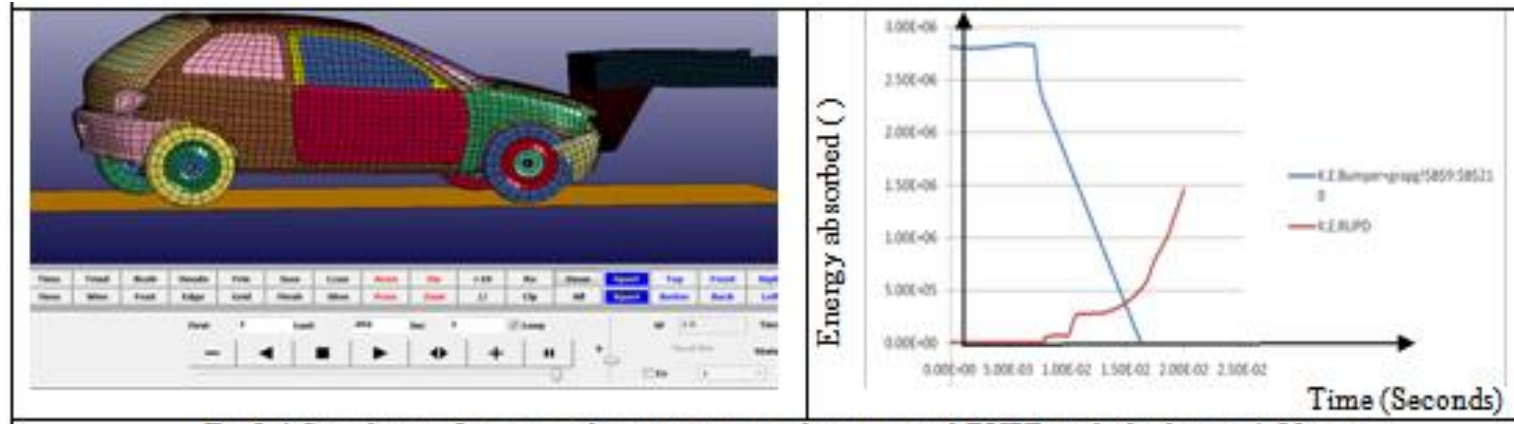

Fig 2.4 Simulation \& energy absorption in car bumper and RUPD with thickness $4.50 \mathrm{~mm}$
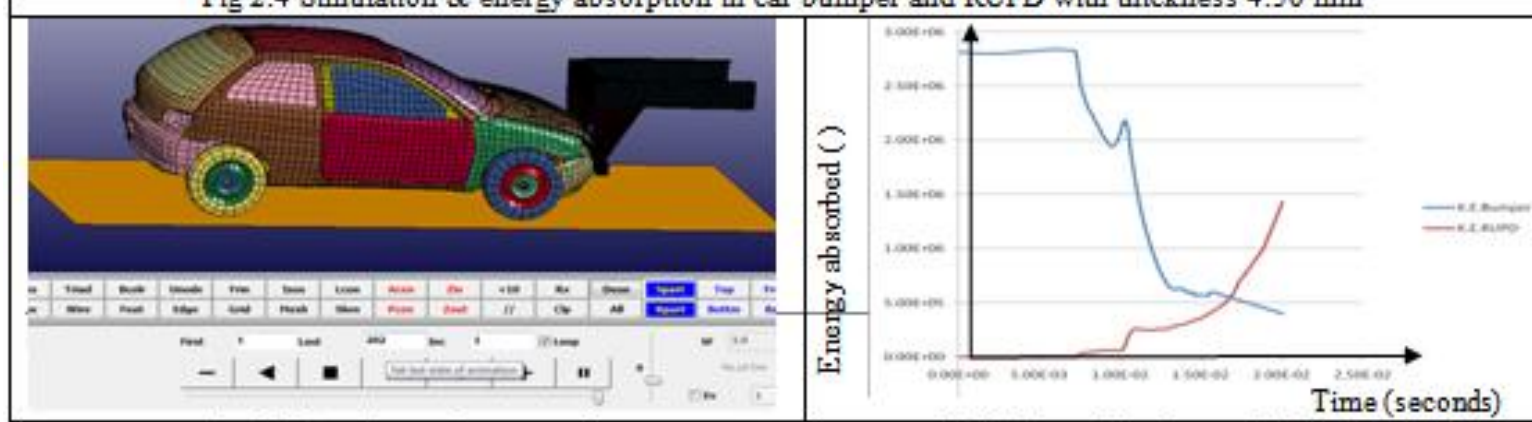

Fig 2.5 Simulation \& energy absorption in car bumper and RUPD with thicknes5 $5.00 \mathrm{~mm}$

\section{RESULTS AND DISCUSSION}

Five Simulation tests were carried out for the rear impact. The model 1 is having $3 \mathrm{~mm}$ thickness of RUPD bar, model 2 is a $3.5 \mathrm{~mm}$ thickness of RUPD, model 3 is a $4 \mathrm{~mm}$ thickness of RUPD, model 4 is a 4.5 mm thickness of RUPD and model 5 is a $5 \mathrm{~mm}$ thickness of RUPD. As observed, the most of the energy of the impact is absorbed by the RUPD bar, bumper and the rails. These components absorb most of the energy of the crash before the tires impacts the rigid bar. The maximum values of kinetic energy of the Test model as shown in graph. For the Test model $2.5 \& 2.6$, whose main purpose was the maximum energy absorption of the RUPD bar of the vehicle, the lower values of the results is not unexpected. The Test model experience lower forces as a result of its weight.

In the case where a vehicle crashes into a rigid fixed RUPD, less stopping distance is required than in braking. The vehicle loses all of its kinetic energy in a fraction of a second through front-end structural deformations. The amount of deformation is equal to the stopping distance of the vehicle. Since the stopping distance of a vehicle in the RUPD crash is normally short, a much higher force is generated at the RUPD interface

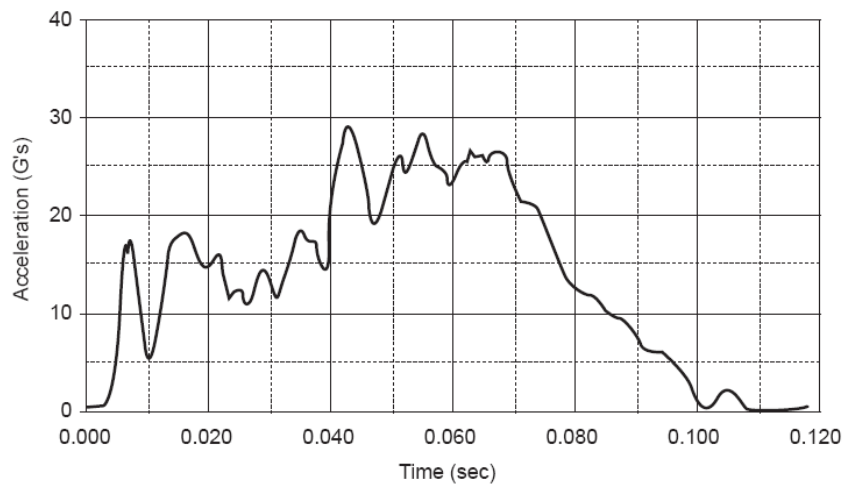

\section{SCOPE OF FUTURE WORK}

The FE model can be used for further simulation of in the simulations of the offset rear impact test, where one side of the rear of the vehicle is impacted against a RUPD or another vehicle. Other tests include the side impact test, where a vehicle is impacted from the side by and oncoming vehicle and oblique car-to-truck or car-to-car impacts the two or more vehicle take part in a collision. Rollover simulation can also be carried out wherein the vehicle rolls on its sides due to the cause of an impact or other factors .

Further crash-testing involving the effects of the crash forces on the occupants of the vehicle can also be carried by using FE models of test dummies. Human- surrogate dummies called Anthropomorphic Test Devices (ATDs) could 
be placed inside the FE vehicle models and an entire crash test event could be simulated. The FE dummies are used to simulate the behavior of a vehicle occupant in the event of a crash. These FE dummies can then be placed inside the vehicle and the crash-simulation performed, they can provide various insights into the dynamic behavior of the human body in the event of a crash. This, however, requires detailed occupant compartment geometry as well as a detailed dummy model. This could easily double the FE models complexity and greatly increase the needed computer resources

\section{CONCLUSION}

The overall objective of the work was to simulate a Rear crash-test and validate the results of the simulations obtained from the crash-test. Simulation was performed using the LS-DYNA software package. The analysis has well established the method and parameters of the simulation on modeling and analysis software. It demonstrates the energy absorption pattern in bumper, rail and RUPD during frontal crash of a car with different design parameters of RUPD. It can be seen from the plots that the RUPD bar absorbs most of the energy during impact of the car bumper. Almost half of the energy of the crash is absorbed by these components after about 0.5 $\mathrm{ms}$ of the crash initiation.

It will be possible to recommend some relevant characteristics for an energy absorbing rear under run protection device. Head on collision contribute significant amount of serious accidents which causes driver fatalities. The car safety performances can work effectively by providing RUPD to the heavy trucks. In India, for Rear Under-run Protection Device, IS 14812:2005 regulation is required in for the trucks to meet the safety requirement to protect under running of the passenger car. In above said design, the maximum displacement of RUPD bar is limited to $50 \mathrm{~mm}$ and the plastic strain is limited to $15 \%$ hence it meet the requirements as per IS 14812:2005. But this needs to be confirmed with physical testing in future. The virtual simulation is a tool which can be used to avoid or reduce the physical testing of mechanical systems and components. Overall effect of this is reduction in development cost as compared to real time physical testing.

\section{REFERENCES}

[1] Z.Q. Chenga, J.G. Thackera, W.D. Pilkeya, W.T. Hollowellb, et.al, "Experiences in reverse- engineering of a finite element automobile crash model", Journal of Finite Elements in Analysis and Design 37 (2001) 843-860.

[2] Abdullatif K. Zaouk, Nabih E. Bedewi, Cing- Dao Kan, et.al, "Validation of a non-linear finite element vehicle model using multiple impact data", The George Washington University FHWA/NHTSA National Crash Analysis Center.

[3] J.O. Hallquist, "LS-DYNA Keyword User_s Manual”, Livermore Software Technology Corporation (1997).

[4] S. W. Kirkpatrick, "Development and Validation of High Fidelity Vehicle Crash Simulation Models", SAE Publications, Presented at the 2000 International Congress and Exposition (2000) SAE Paper No. 00PC-248.

[5] Computational approval of rear under run protection device with MSC.nastran

[6] Prof. Dr. Zoran Ren University of Maribor, Faculty of Mechanical Engineering

[7] Bjornstig J, Bjornstig Ulf, Eriksson A, "Passenger car collision fatalities - With special emphasis on collision with heavy vehicles", Accident Analysis and Prevention 2008, P 158-166.

[8] Vehicle Standard (Indian Automotive Standard) for Rear Under-run Protection Device IS 14812 - 2005

[9] Langner, W," The ODB test, a challenge to vehicle construction”, SAE 967031

[10] Cook et al., "Concepts and Applications of Finite Element Analysis", John Wiley \& Sons, 1989

[11] Xiao Lin, "Numerical Computation of Stress Waves in Solids", John Wiley \& Sons, 1996.

[12] Den Hartog, “Advanced Strength of Materials”, pp 193, Dover, 1987. 